\title{
Cascaded uncoupled dual-ring modulator
}

\author{
Tingyi Gu, ${ }^{1,2}$ Young-Kai Chen, ${ }^{1}$ Chee Wei Wong, ${ }^{2}$ and Po Dong ${ }^{1, *}$ \\ ${ }^{1}$ Bell Labs, Alcatel-Lucent, 791 Holmdel Road, Holmdel, New Jersey 07733, USA \\ ${ }^{2}$ Optical Nanostructures Laboratory, Columbia University, New York, New York 10027, USA \\ ${ }^{*}$ Corresponding author: po.dong@alcatel-lucent.com
}

Received June 26, 2014; accepted July 17, 2014;

posted July 21, 2014 (Doc. ID 214817); published August 15, 2014

\begin{abstract}
We demonstrate, by coherent driving two uncoupled rings in same direction, that the effective photon circulating time in the dual-ring modulator is reduced, with increased modulation quality. The inter-ring detuning-dependent photon dynamics, $Q$ factor, extinction ratio, and optical modulation amplitude of two cascaded silicon ring resonators are studied and compared with that of a single-ring modulator. Experimentally measured eye diagrams, together with coupled mode theory simulations, demonstrate the enhancement of the dual-ring configuration at 20 Gbps with a $Q \sim 20,000$. (c) 2014 Optical Society of America

OCIS codes: (130.0130) Integrated optics; (140.4780) Optical resonators; (130.4110) Modulators.

http://dx.doi.org/10.1364/OL.39.004974
\end{abstract}

Silicon photonics modulators have emerged as low-power optical transmitters in combination with energy-efficient CMOS driver amplifiers using hybrid electronic-photonics integration or monolithic integration [1-5]. The silicon modulators were also integrated into an intrachip wavelength division multiplexing (WDM) optical link to form a reconfigurable switch fabric for chip-level optical interconnects and network []. pactness and low energy consumption, silicon microring/ microdisks are identified as one of the most energyefficient photonic modulators. High-speed modulation of silicon microrings could be obtained by modulating the reverse-biased $p n$ junctions embedded in their waveguides. Compared to electrical delay, the photon lifetime usually sets the upper limit of the cavity modulation rate $[\underline{4}, 7-9]$. To reduce the cavity lifetime for higher modulating speed, several schemes were investigated with complex design and layouts. For example, the modulation speed was improved by two times utilizing the couplingmodulated microrings $[10,11]$. However, these approaches result in expanded device footprint and require nonconventional fabrication sequences. Dual resonator configuration, such as two rings with a feedback loop or coupled photonic crystal cavities, have been also used to engineer the effective photon lifetime [12-14], in combination with thermo-optic detuning controls $[\overline{15}, 16]$. In this Letter we show that, with a conventional fabrication process and compact device layout, the modulator bandwidth can be improved by introducing cascaded microrings without any feedback. By directing input photons into two microring cavities in series, the resultant effective photon lifetime in the two-ring system can be reduced by half, thereby improving the modulation bandwidth.

Photon dynamics in cascaded uncoupled rings can be described by time domain coupled mode theory (CMT). The photon amplitudes in the first and second resonators are

$$
\begin{gathered}
\frac{d a_{1}}{d t}=\left(i\left(\omega_{1}-\omega\right)-\frac{1}{2 \tau_{1}}\right) a_{1}+\sqrt{\frac{1}{2 \tau_{1 c}}} s_{1+} \\
\frac{d a_{2}}{d t}=\left(i\left(\omega_{2}-\omega\right)-\frac{1}{2 \tau_{2}}\right) a_{2}+\frac{1}{2 \tau_{2 c}} e^{i(-\beta L)} a_{1}+\sqrt{\frac{1}{2 \tau_{2 c}}} e^{i(-\beta L)} s_{1+},
\end{gathered}
$$

where $a_{1}$ and $a_{2}$ are light amplitudes in the first and second rings. The total photon lifetime $\left(\tau_{1(2)}\right)$ in a single resonator is $1 / \tau_{1(2)}=1 / \tau_{1(2) c}+1 / \tau_{1(2) \text { in }}$, where $1 / \tau_{c}$ and $1 / \tau_{\text {in }}$ are coupling and intrinsic loss rates, respectively. $\omega_{01}$ and $\omega_{02}$ represent the cavity-resonant frequencies which are dynamically tunable by carrier depletion to be time dependent as $\omega_{1}=\omega_{01}+\Delta \omega$ and $\omega_{2}=\omega_{02}+\Delta \omega$, respectively. $\omega$ is the frequency of input laser, and $\Delta \omega$ is the resonance shifted by carrier depletion modulation. $\beta$ is the propagation constant in the waveguide and $L$ is the length of the waveguide between two rings. $s_{1+}=\operatorname{sqrt}\left(P_{\text {in }}\right)$ is the external optical power supply term. The second terms in Eq. (1) and third term in Eq. (2) represent its coupling to the first and second resonator, respectively. The second term in Eq. (2) describes photon coupling from the first resonator to the second, one with phase delay in waveguide $(\beta L)$.

Figures 1(a) and 1(b) exhibit the transmission spectra of a single (blue) and dual-ring (red) modulator. Two ring resonators are coupled to the same waveguide without a feedback loop [inset of Fig. 1(a)]. Here, we set single-ring resonators with 20,000/80,000 coupling/intrinsic quality factor, and minimal transmission of $-14 \mathrm{~dB}$. Intrinsic quality factor (Qin) is determined by the propagation loss of the waveguide, while coupling quality factor $(Q c)$ depends on the ring-waveguide coupling gap. Both of the quality factors are predetermined by materials and nanofabrication. The total quality factor $(1 / Q t=1 / Q \mathrm{in}+$ $1 / Q c)$ is proportional to the photon lifetime $(\tau=Q t / \omega)$. The ratio between $Q$ in and $Q c$ sets the upper limit of the extinction ratio (ER) for the single ring [17]. For a singlering modulator, $Q c$ can be varied in the design to compromise between ER and photon lifetime. With the given $Q s$, the ER of a single-ring modulator is about $7.9 \mathrm{~dB}$, as the electrical signal drives the resonance shift to about half of the full wave half-maximum (FWHM). ER is greatly enhanced by the dual-ring modulator to $20.1 \mathrm{~dB}$, with zero inter-ring detuning, at the same laser resonance detuning as a single-ring modulator. However, the enhanced insertion loss brings an extra power penalty and may degrade optical modulation amplitude (OMA). The OMA can be separately optimized by a dual-ring configuration, with inter-ring detuning set as $60 \mathrm{pm}$ [Fig. 1(b)]. The OMA and ER of a dual-ring modulator can be separately optimized at different inter-ring detunings [Fig. 1(c)]. 

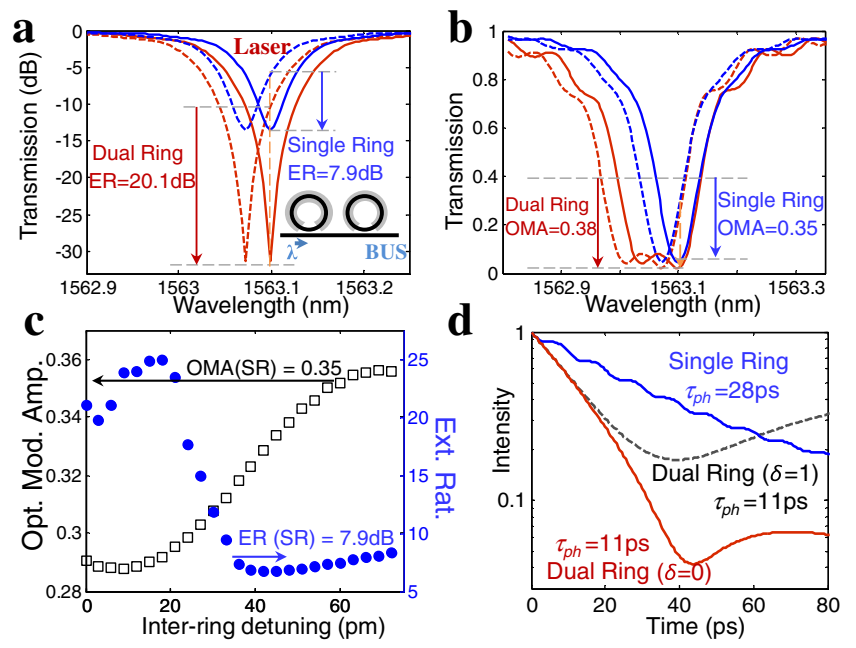

Fig. 1. Spectral and time domain schematics of a dual-ring modulator. (a) Transmission spectrum of typical single- (blue) and dual-ring (red) modulator, with typical $Q(s)$ of 20,000 . The initial state at zero reverse bias (dashed curves) are shifted $\sim 30 \mathrm{pm}$ to the right (solid lines). Inset, schematics of dual-ring modulator coupled to the same bus waveguide. (b) Transmission spectrum of single- and dual-ring modulator with $60 \mathrm{pm}$ inter-ring detuning. (c) OMA and ER versus inter-ring detuning for a dual-ring modulator, where the OMA and ER of the single ring are marked by arrows. (d) Photon decay dynamics for single and dual ring.

Quality factors decide the photon lifetime circulating in the ring. As a pulse excitation (delta function) is sent into the waveguide at $0 \mathrm{ps}$, the transmission decay dynamics for single- (blue curves) and double-ring configuration (red solid curve) are given in Fig. 1(d). Light circulates in a ring for the photon lifetime of $28 \mathrm{ps}$, and drops to $\sim 11 \mathrm{ps}$ when the two rings are coherently driven [Fig. 1(d)]. The effective photon lifetime of a dual-ring modulator is insensitive to inter-ring detunings. If the resonance of the two rings are not well aligned (by thermo-optic tuning), a long tail would degrade the ER and thus the output eyes, while the photon lifetime maintains same as the zero detuning case [dashed curve in Fig. 1(d),$\delta=\left(\lambda_{\text {ring2 }}-\lambda_{\text {ring2 }}\right) /$ $(\Delta \lambda / 2)=1$ ]. It shows that the response time for the dualring modulator has high tolerance for resonance offset between the two rings. Slightly different intrinsic quality factors between the two rings leads to the ripples on the tail, and can be eliminated when the quality factors for the two rings are identical.

The reduced effective coherently modulated photon lifetime would lead to better high-speed modulation quality. The initial laser resonance detuning is set as $30 \mathrm{pm}$. Given the quality factors for the two rings combined with random pattern input setting, CMT describes the ideal eye diagram for single-ring [Fig. 2(a)] and doublering [Fig. 2(b)] with push-push scheme $\left[\delta_{\text {Mod1 }}=\Delta \lambda /\right.$ $\left(\Delta \lambda_{\text {Bandwith1 } 1 / 2)}=1.5, \delta_{\text {Mod } 2}=1.5\right.$, Fig. $\left.4(\mathrm{~b})\right]$ at $40 \mathrm{~Gb} / \mathrm{s}$ modulation speed. Dual-ring gives the better eye opening [numerically expressed as Qfactor $=\left(P_{1}-P_{0}\right) /\left(\sigma_{1}+\right.$ $\left.\sigma_{0}\right)$ ]. From the histogram on the eye width, the power level $\left(P_{1 / 0}\right)$ and root mean squares $\left(\sigma_{1 / 0}\right)$ for one and zero levels can be fit by a Gaussian. Here, the simulation does not include the electrical resistance-capacitance times, which in practice would limit the modulation speed as well.

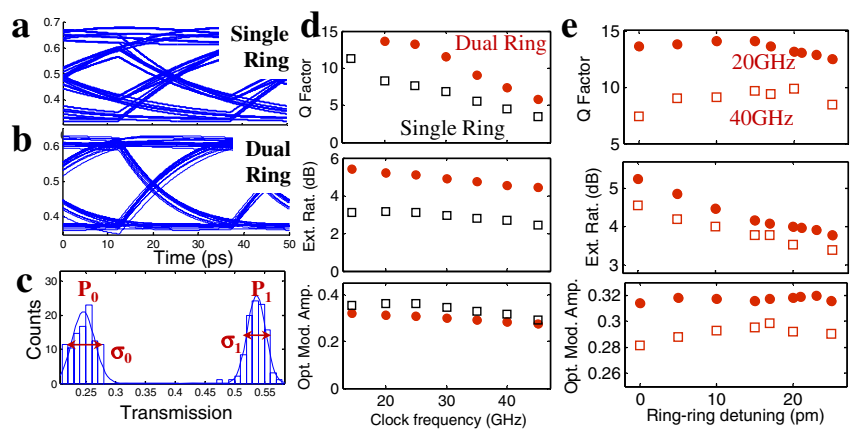

Fig. 2. High-speed modulation performance of a dual-ring modulator. (a) Simulated modulation eye diagram for single ring. (b) Double-ring operation at $40 \mathrm{~Gb} / \mathrm{s}$. (c) Histogram of the transmission along the eye height. (d) $Q$ factor, ER, and OMA of a dual-ring (red solid circle) and single-ring (black empty square) modulator with zero inter-ring detuning. (e) Inter-ring detuning for a dual ring operating at $20 \mathrm{GHz}$ (solid circles) and $40 \mathrm{GHz}$ (empty squares).

The "eye opening" decreases as the clock frequency increases. The dual-ring modulator excels in terms of $Q$ factor and ER over the single-ring for simulated clock frequency ranges from 15 to $45 \mathrm{GHz}$ at zero inter-ring detunings. The increased insertion loss slightly lowers the OMA for the dual-ring modulator [Fig. 2(d)]. The inter-ring detuning is also optimized at 20 and $40 \mathrm{GHz}$ clock frequencies, as shown in Fig. 2(e).

To verify the speed enhancement, we use a silicon photonic integrated circuit including 10 ring modulators coupled to a bus waveguide, which was originally used in [6]. The chip was packaged with two fibers with a fiberto-fiber loss of $13 \mathrm{~dB}$ [Fig. 3(c)]. In the current study, we randomly pick 2 adjacent rings out of the 10 . The ring resonance is slowly tuned close to laser resonance and fixed by integrated heaters. The data drive is simultaneously applied to both rings through dual $\mathrm{RF}$ probes [Fig. 3(a)]. The resonance can be tuned by a microheater on top of the ring, and the $p n$ diodes crossing each ring can quickly modulate the ring resonance [Fig. 3(b)]. By placing the heater right on top of the waveguide, the Ohm

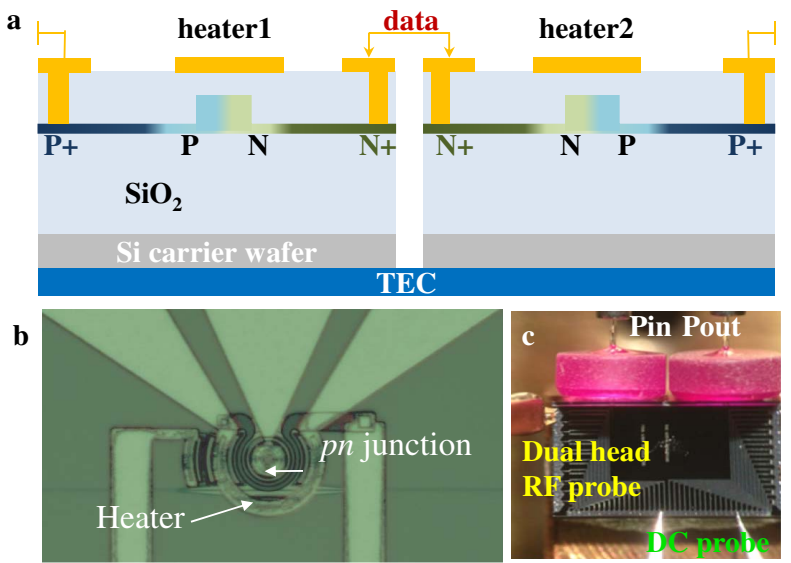

Fig. 3. Device layout. (a) Schematic cross-section drawing of dual-ring modulator with lateral $p n$ junction and integrated heaters. (b) Optical top view of the silicon ring with integrated $p n$ junction and heater. (c) The devices under test are reconfigurable photonic networks-on-chip-based microrings. 
heat diffuses through the oxide layer to the silicon waveguide, and effectively adjusts the laser cavity detuning.

The steady-state characterizations of the rings (transmission and delay) are preceded by a Luna Technologies optical vector analyzer [Fig. 4(a)] [18], and the measurements are fitted by CMT. The red circles are experimental data and black lines are theoretical fitting. By fitting the transmission and delay spectrum, the coupling/intrinsic quality factors for the two rings are 20,000/85,000 and 22,000/100,000, respectively [Fig. 4(a)]. The thermal tuning and electro-optic modulation (reverse bias) are measured to be 80.5 and $22 \mathrm{pm} / \mathrm{V}$ respectively, as summarized from 10 ring modulators with integrated heater and $p n$ junctions in [].

The modulation quality (eye opening) is electrically limited by two factors: electro-optic modulation depth and the electrical resistance-capacitance constant (electrical delay). As the electrical delay is given by the $p n$ junction fabrication, we focus on optimizing the reverse-biasdependent transmission modulation and correspondent laser resonance detunings, where the peak-to-peak driving voltage $\left(V_{p p}\right)$ is quadratically related to energy consumption per bit [19]. Figure $\underline{4(b)}$ measures the transmission spectrum of the two rings under reverse bias from 0 to $-3 \mathrm{~V}$, with $1 \mathrm{~V}$ per step. The transmission dip (resonance) is effectively shifted from zero detuning $(\delta=0)$ to $\delta=$ -1.4 [lower-right inset of Fig. 4(b)]. The carrier-densitydependent linear loss modifies the on-resonance transmission near the critical coupling, as the reverse bias redshifts the resonance [20,21]. The minor modification of ER would not affect our conclusions. The tuning is most efficient as the reverse bias increases from 0 to $-2 \mathrm{~V}$, and we chose this region as the modulation range. The tuning efficiency saturates at higher bias range. A simple quadratic fit for the measured resonance versus reverse bias plot gives $\left(\lambda_{\text {laser }}-\lambda_{\text {res }}\right)=-4.5 \mathrm{~V}^{2}+31 \mathrm{~V}+0.13 \mathrm{pm}$. Given the ring radius of $15 \mu \mathrm{m}$, the $\mathrm{RC}$ constant for the single-ring modulator is estimated to be $\sim 8 \mathrm{ps}$. For the dual-ring modulator, the parallel connected pn junctions with halfresistance and double capacitance keep the same RC constant as single ring.

The high-speed measurement was carried out using a dual-head RF probes and two DC probes [Fig. 3(c)]. The resonances of the two neighboring rings are independently
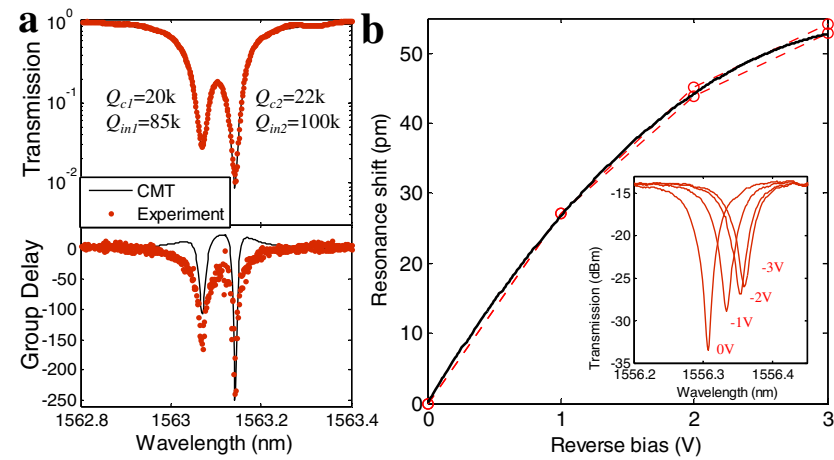

Fig. 4. DC characteristics. (a) Transmission and delay measurement (red dots) and CMT simulation of double rings. (b) Resonance shift versus the reverse-biased voltage. The dots are experimental data and the black line is quadratic fit. Inset, transmitted power versus normalized laser resonance detuning of the ring under reverse bias from 0 to $-3 \mathrm{~V}$. tuned to be close to the laser wavelength at $1563 \mathrm{~nm}$ by the integrated heaters, with $30 \mathrm{pm}$ detuning between the ring resonance and laser wavelength. The $0-2 \mathrm{~V}$ reverse bias modulates ring resonance shifting another $30 \mathrm{pm}$ away from laser wavelength. The high-speed performance is measured by optical eye diagrams at a $20 \mathrm{~Gb} / \mathrm{s}$ rate.

The high-speed $(20 \mathrm{~Gb} / \mathrm{s})$ operations for the first and second rings are recorded on sampling oscilloscope [Figs. 5(a) and 5(c), with 20 ps per grid]. The ERs for the eye diagrams are 5.9 and $5.8 \mathrm{~dB}$ for single-ring operation, and are enhanced to $7.13 \mathrm{~dB}$ with double-ring cooperation [Fig. 5(e)], with optimized thermo-optic detunings. Correspondent simulations by CMT with the parameters measured in steady state are presented in Figs. 5(b), 5(d), and 5(f) for comparison. The eye opening was enhanced as well, matching the theoretical prediction that the fall/rise times are shortened due to reduced effective photon lifetime by coherent modulation.

We have demonstrated that a cascaded dual-ring modulator without feedback can enhance the high-speed modulation quality for microring modulators. The simple decay dynamics predict enhanced photon loss rate when two neighboring identical rings are coherently modulated by the same electrical signal. Experimental demonstration of better eye opening at 20 Gbps operation for the double-ring is performed by adjusted detuning on the reconfigurable silicon photonic network-on-chip platform, which agrees theoretically with the CMT calculations.

Part of this project is funded by the Intelligence Advanced Research Projects Agency (IARPA) under SPAWAR contract number N66001-12-C-2011. The views and conclusions contained herein are those of the authors and should not be interpreted as necessarily representing the official policies or endorsements, either
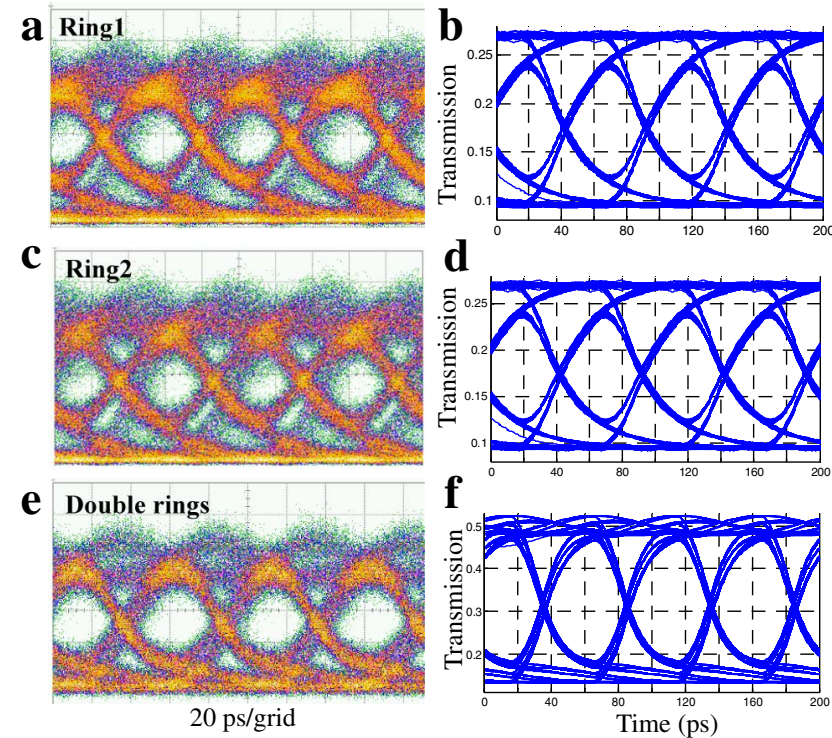

Fig. 5. Comparison of $20 \mathrm{~Gb} / \mathrm{s}$ operation for single- and dualring modulators. (a) Measured eye diagrams on the output port of ring 1 on sampling oscilloscope. (b) Time domain CMT simulation of superposition of 200 single shots, with pseudorandom input. (c) Experimental and (d) correspondent CMT calculation for ring 2. (e) Experimental and (f) CMT calculation for double rings with synchronized electrical data drive (considering $10 \mathrm{ps}$ electrical delay). 
expressed or implied, of ODNI, IARPA, or the U.S. Government. The U.S. Government is authorized to reproduce and distribute reprints for governmental purposes notwithstanding any copyright annotation thereon. We acknowledge the support of Dr. Dennis Polla at IARPA, Drs. T.-Y. Liow and Patrick G.-Q. Lo of the Institute of Microelectronics, Singapore, on fabrication. T. Gu acknowledges discussions with X.-L. Zhu and C. Chen from Prof. K. Bergman's group at Columbia University, and W. M. J. Green from IBM. T. Gu and C. W. Wong acknowledge partial support from NSF (1069240).

\section{References}

1. P. Dong, W. Qian, H. Liang, R. Shafiiha, D. Feng, G. Li, J. E. Cunningham, A. V. Krishnamoorthy, and M. Asghari, Opt. Express 18, 20298 (2010).

2. X. Zheng, D. Patil, J. Lexau, F. Liu, G. Li, H. Thacker, Y. Luo, I. Shubin, J. Li, J. Yao, P. Dong, D. Feng, M. Asghari, T. Pinguet, A. Mekis, P. Amberg, M. Dayringer, J. Gainsley, H. F. Moghadam, E. Alon, K. Raj, R. Ho, J. E. Cunningham, and A. V. Krishnamoorthy, Opt. Express 19, 5172 (2011).

3. M. R. Watts, W. A. Zortman, D. C. Trotter, R. W. Young, and A. L. Lentine, Opt. Express 19, 21989 (2011).

4. A. Liu, L. Liao, D. Rubin, H. Nguyen, B. Ciftcioglu, Y. Chetrit, N. Lzhaky, and M. Paniccia, Opt. Express 15, 660 (2007).

5. V. M. Passaro and F. Dell'Olio, IEEE Trans. Nanotechnol. 7, 401 (2008).

6. P. Dong, Y. K. Chen, T. Gu, L. L. Buhl, D. T. Neilson, and J. H. Sinsky, in Optical Fiber Communication Conference (Optical Society of America, 2014), paper Th4G.2.

7. A. C. Turner-Foster, M. A. Foster, J. S. Levy, C. B. Poitras, R. Salem, A. L. Gaeta, and M. Lipson, Opt. Express 18, 3582 (2010).
8. N.-N. Feng, S. Liao, D. Feng, P. Dong, D. Zheng, H. Liang, R. Shaffiha, G. Li, J. E. Cunningham, A. V. Krishnamoorthy, and M. Asghari, Opt. Express 18, 7994 (2010).

9. F. Y. Gardes, A. Brimont, P. Sanchis, G. Rasigade, D. MarrisMorini, L. O'Faolain, F. Dong, J. M. Fedeil, P. Dumon, L. Vivien, T. F. Krauss, G. T. Reed, and J. Marti, Opt. Express 17, 21986 (2009).

10. W. D. Sacher, W. M. J. Green, S. Assefa, T. Barwicz, H. Pan, S. M. Shank, Y. A. Vlasov, and J. K. S. Poon, Opt. Express 21, 9722 (2013).

11. T. Gu, C. W. Wong, Y. Chen, and P. Dong, in CLEO: Science and Innovations (Optical Society of America, 2014), paper SM2G-1.

12. Q. Xu, P. Dong, and M. Lipson, Nat. Phys. 3, 406 (2007).

13. S. Kocaman, X. Yang, J. F. McMillan, M. B. Yu, D. L. Kwong, and C. W. Wong, Appl. Phys. Lett. 96, 221111 (2010).

14. Y. Hu, X. Xiao, H. Xu, X. Li, K. Xiong, Z. Li, T. Chu, Y. Yu, and J. Yu, Opt. Express 20, 15079 (2012).

15. C. Qiu, J. Shu, Z. Li, X. Zhang, and Q. Xu, Opt. Express 19, 5143 (2011).

16. T. Gu, S. Kocaman, X. Yang, J. F. McMillan, M.-B. Yu, G.-Q. Lo, D.-L. Kwong, and C. W. Wong, Appl. Phys. Lett. 98, 121103 (2011).

17. Y. Akahane, T. Asano, B.-S. Song, and S. Noda, Opt. Express 13, 1202 (2005).

18. D. K. Gifford, B. J. Soller, M. S. Wolfe, and M. E. Froggatt, Appl. Opt. 44, 7282 (2005).

19. P. Dong, S. Liao, D. Feng, H. Liang, D. Zheng, R. Shafiiha, C.-C. Kung, W. Qiang, G. Li, X. Zheng, A. V. Krishnamoothy, and M. Asghari, Opt. Express 17, 2248 (2009).

20. A. Yariv, IEEE Photon. Technol. Lett. 14, 483 (2002).

21. H. Y. Wen, O. Kuzucu, M. Fridman, A. L. Gaeta, L.-W. Luo, and M. Lipson, Phys. Rev. Lett. 108, 223907 (2012). 